\title{
DU DÉVELOPPEMENT DE LA MICROFILAIRE DU GYMNODACTYLUS FEDSCHENKI
}

\author{
Par le professeur E. MARZINOWSKY \\ Directeur de l'Institut tropical de Moscou
}

En 1913, Delanoë a décrit des filaires dans le foie d'Agama colonorum, et en même temps des microfilaires dans le sang dı même animal, avec le cycle évolutif complet. Les femelles adultes étaient de couleur blanc-laiteux et mesuraient de 12 à 13 centimètres de long sur $0 \mathrm{~cm}$., 275 de large. Les mâles n'ont pas été observés.

Cet auteur avait vu les microfilaires dans le sang pendant environ un mois et leur quantité était à peu près la même durant le jour et durant la nuit. Ces microfilaires étaient incluses dans des gaines et avaient la possibilité de se déplacer librement à l’intérieur. Delanoë avait également observé des gaines vides. Les microfilaires mesuraient environ $190 \mu$ de long sur $6 \mu$ de large ; elles présentaient des stries transversales distinctes. Les noyaux embryonnaires étaient disposés sur deux ou trois rangs, sauf à l'extrémité postérieure, où ils étaient placés sur un seul rang. Les taches n'étaient pas toujours très visibles, surtout la tache céphalique qui, souvent, était à peine distincte. La tache caudale était toujours bien marquée et mesurait de 6 à $8 \mu$ de large.

On a trouvé en outre dans le sang du foie des œufs de ces filaires à différents stades de développement et, en même temps, des cellules particulières que Delanoë considère comme des œufs atrophiques.

En 1916, nous avons découvert à Enseli, en Perse, sur des frottis de sang périphérique et dans les organes internes de Gymnoaactylus fedschenki Strauch, un grand nombre de microfilaires, qui se distinguaient par leurs dimensions de celles qu'avait décrites Delancë ; elles mesuraient en effet $102 \mu$ de long sur $8 \mu$ de large. Sur des frottis de foie, nous avons trouvé en outre des cellules très granuleuses avec noyau- excentrique, qui ne pouvaient être autre chose que des œufs de filaires. Ces mêmes cellules se retrouvaient, mais plus rarement, dans le sang périphérique. A l'intérieur ou à ia surface de la plupart de ces œufs, on remarque de petits vermicu-

Annales de Parasitologie, $\mathrm{T} . \mathrm{V}, \mathrm{N}^{\circ} 2$. $-1^{\mathrm{er}}$ avril 1927 , p. 114-116. 
les qui sont le plus souvent recourbés et que nous ne sommeś jamais parvenus à colorer.

Nous avons aussi observé d'autres éléments qui avaient l'aspect de microfilaires typiques, pliées en deux, parfois redressées, mais toujours renfermées dans une gaine homogène colorée en rose clair. Les gaines mesurent pour la plupart $114 \mu$-de long sur 9 à $10 \mu$ de large ; elles sont par conséquent plus longues et plus larges que les microfilaires elles-mêmes, ce qui permet à ces dernières de se mouvoir à l'intérieur, ainsi que nous l'avons observé à l'examen de sang frais. Parfois, la gaine entoure de près la microfilaire
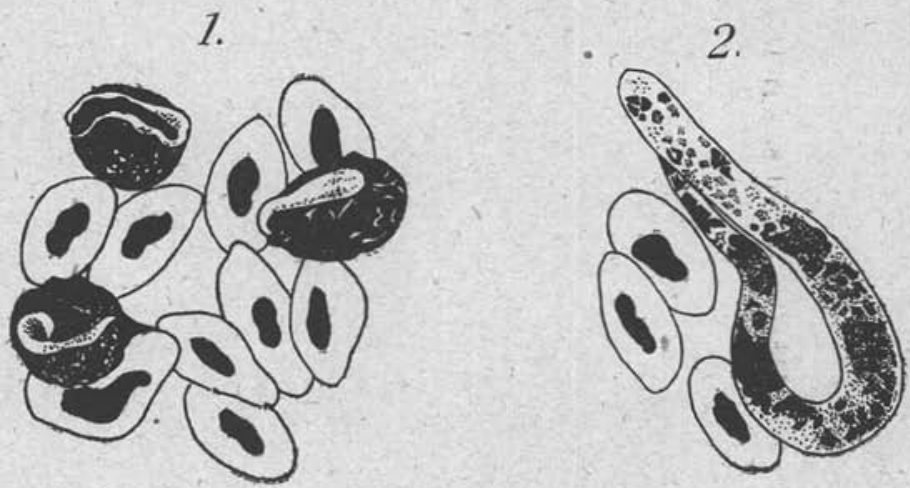

Fig. - 1, Évolution des microfilaires dans les œufs (frottis de foie). 2, Microfilaire dans un frottis de foie.

et est difficile à distinguer nettement. On peut considérer ces formes comme des microfilaires adultes.

Il existe une grande quantité de noyaux embryonnaires qui sont répartis dans tout le corps, même à l'extrémité caudale, où ils sont disposés sur un ou deux rangs. La tache céphalique est bien marquée chez presque tous les exemplaires; la tache oblique et la tache en forme d'U sont moins distinctes, surtout la dernière, bien que toutes deux soient visibles sur la plupart des exemplaires. Le corpuscule central de Manson se voit distinctement, ainsi que la tache caudale. L'anneau nerveux est situé à $14 \mu$ de l'extrémité céphalique. La cellule excrétrice, qui mesure $7 \mu$,' est située à $6 \mu$ de l'extrémité caudale. La première cellule génitale mesure $9 \mu$ et est située à $26 \mu$ de l'extrémité antérieure. Un peu plus loin, à une distance de $3 \mu$, se trouve la seconde cellule gênitale qui a les mêmes dimensions que la première et, plus loin encore, se trouve 
la troisième cellule génitale qui mesure $11 \mu$. Le pore génital, d'un diamètre de $3 \mu$, se trouve placé à $36 \mu$ de l'extrémité antérieure.

Nous n'avons pas réussi à trouver de filaires adultes, ce qui tient probablement à la petite quantité de lézards que nous avons disséqués. Toutefois, ëtant donné le grand nombre d'œufs que l'on observe dans le foie, on peut admettre que les filaires adultes occupent cet organe, ce qui s'accorde bien ayec les observations de Delanoë.

Rodhain, en 1906, a décrit chez les agames, dans le tissu adipeux sous-cutané, des filaires qui se distinguent nettement de celles qu'a décrites Delanoë par leurs plus petites dimensions ; ces filaires mesurent $66 \mu$ de long, la longueur de la gaine étant de $89 \mu$.

Quant aux microfilaires que nous avons trouvées, elles ressemHent beaucoup à celles qui ont été décrites par Delanoë (1).

(1) Detanof (P.). - Au sujet de l'existence chez un saurien, Agama colonornm Dum. et Bibr. d'une filalre et d'une microfilaire sanguines. Buill. soc. pothol. exot., VII, 1914, p. 121. 